\title{
Interanimal negative transfer of the kindling effect*
}

\author{
JOHN GAITO and STEPHEN T. GAITO \\ York University, Downsview 463, Ontario, Canada
}

\begin{abstract}
Five experiments were conducted in which donor rats were kindled to the clonic-convulsion stage, sacrificed, and their brains removed. The brain was homogenized, and the supernatant fraction was injected intraperitoneally into recipient experimental rats, who then were subjected to the kindling procedure. Control donors which received no stimulation were included. When the injection involved two or more brain amounts, a retarding effect tended to occur with the experimentals. If only one brain amount was used for the injection, no change resulted in the kindling rate of these recipients. This interanimal negative-transfer effect appears to be similar to the intraanimal negative-transfer effect reported by McIntyre and Goddard.
\end{abstract}

In the last 20 years there have been attempts by many investigators to determine the brain neurochemical events which occur during learning behavior (Gaito, 1966, 1971). Molecular Psychobiology Laboratory personnel at York University have been engaged in this task for approximately a decade (Gaito, 1972). A major problem in these attempts has been that of ensuring that the control or "nonlearning" animal does not show learning so as to contrast sharply with the "learning" animal. Even though the control animal does not learn the task which the experimental animal is subjected to, he may be learning other things. Another problem is that learning behavior is influenced greatly by motivation and other extraneous aspects. Thus, it would be more efficacious to choose a behavioral paradigm which is similar to learning, but in which control conditions are more adequate. One possibility is that of the "kindling effect."

A group of researchers (Goddard, et al, 1969; McIntyre, 1970; McIntyre \& Goddard, 1973) have found that repeated low-intensity stimulation, via implanted electrodes in a number of subcortical sites, eventually leads to bilateral clonic convulsions. For example, electrical stimulation administered daily to the amygdala produces clonic convulsions, on the average, in about 10 to 15 trials. The initial stimulations have little effect on the animal's behavior (Stage 1-normal exploratory behavior); with a few repetitions, overt indications of seizure activity can be observed, e.g., eye closure, chewing, salivation [Stage 2-behavioral automatisms (BA)]. With further stimulations, these automatisms culminate in a complete convulsion [Stage 3-clonic convulsions (CC)]. Although certain behaviors during Stage 2, such as eye closure and chewing, are relatively stereotyped and easy to identify, other behaviors can be confused with those observed during Stage 1 (e.g., arrest, head turning to ipsilateral side). However, the clonic convulsions encountered in Stage 3 are relatively the

*We thank Olive and Donna Gaito for assistance in the construction of bipolar electrodes. This research was supported by the President's NRC Fund (Grant 32) from York University and a grant from the Ontario Society for Crippled Children. same from animal to animal. The rat stands on its hind paws and bilateral clonic convulsions ensue; these continue after the electrical stimulation is terminated. Thus Stage 3 behavior is less variable, and easier to identify, than that of Stage 2.

Attending these behavioral changes are neurophysiological modifications (Racine, 1972). For example, the following changes occur upon stimulating the amygdala: First, there is the normal electrical pattern. With repeated stimulation, the threshold for an after discharge (AD) is decreased. Soon the AD appears; it is a simple spike wave form, $1 \mathrm{~Hz}$, with a duration of about $6-50 \mathrm{sec}$, and a mean amplitude of $702 \mathrm{mV}$. The first AD appears before behavioral automatisms are observed. Upon further stimulation, the wave form becomes more complex, and increases occur in frequency, duration, and amplitude. Then an $\mathrm{AD}$ is induced in the contralateral amygdala. At first, the amplitude of this wave is lower than the AD in the ipsilateral amygdala. When the two are approximately equal in amplitude, clonic convulsions ensue.

The kindling paradigm was used in the present study. The purpose of this study was to determine if the supernatant of homogenized brain from kindled rats would facilitate or impair kindling, when injected into naive recipients. Results of a preliminary study suggested that a retarding effect was present (Gaito et al, 1973).

\section{METHODS AND RESULTS}

Prior to the initiation of formal experiments, brain coordinates similar to those of McIntyre (1970) $(.5 \mathrm{~mm}$ posterior to bregma, $4.5 \mathrm{~mm}$ from midline, $8.5 \mathrm{~mm}$ from skull) were utilized with a number of animals. Brain slices were prepared and stained to determine the location of the implanted electrodes. These histological analyses indicated that the tip of the electrode was in the amygdala. Thus, all experiments utilized these specific coordinates.

In all experiments, Wistar-strain male rats (90 to 130 days old at the beginning of the experiment) had bipolar electrodes (Nichrome wire with trimel coating, $.127 \mathrm{~mm}$ 
in diam, dipped once in Epoxylite) implanted into the amygdala. Half of the animals had electrodes inserted into the right hemisphere-the others, into the left hemisphere. All electrodes were inspected for electrical adequacy before insertion and after sacrificing of the rat. All recipients were coded and run by an experimenter who was unaware of the designation of each rat.

\section{Experiment 1}

Goddard et al (1969) indicated that stimulation of one amygdala facilitated kindling in the other amygdala (intraanimal positive transfer, facilitation), but that rekindling of the first amygdala was then impaired (intraanimal negative transfer, retardation). This experiment was concerned with the possibility of interanimal transfer of positive or negative nature.

Thirty-four donor rats, from six litters, had electrodes implanted unilaterally in the amygdala. Six or more days later, the kindling procedures began. Twenty-four experimental rats (E) were stimulated at approximately $12-\mathrm{h}$ intervals by a $60-\mathrm{Hz}$ sine wave of 100 microA intensity (peak-to-peak) for $60 \mathrm{sec}$ from a Lafayette sine-wave stimulator. A response was scored as a clonic convulsion only if the convulsion continued after termination of the current. On the tenth $\mathrm{CC}$ trial, at $10 \mathrm{~min}$ after the initiation of the trial, the rats were sacrificed by decapitation. The brain was rapidly removed; the olfactory bulbs, cerebellum, and brainstem parts below the cerebellum were deleted; the remainder was used for injections. Each brain was inspected visually to determine the position of the electrode. This was accomplished by two cuts: a vertical slice at the site at which the electrode entered the brain, perpendicular to the longitudinal fissure, and a similar one along the longitudinal fissure. Then the anterior portion of the hemisphere was removed to provide an excellent coronal view of the electrode location in both the removed (anterior) portion and the intact (posterior) portion. The electrode made a thin, easily discerned, line from the point of entry to its location, subcortically. The brains were stored at $-60^{\circ} \mathrm{C}$ until used.

Ten controls (C), littermates of the $\mathrm{E}$ rats, were connected to stimulator lead wires but received no stimulation. The Cs were sacrificed and treated in the same manner as the Es.

Two $\mathrm{E}$ brains were homogenized together in physiolngical saline. A similar procedure was used with $\mathrm{C}$ brains. The homogenates were shaken overnight at $2^{\circ} \mathrm{C}$ and centrifuged at $20,000 \mathrm{xg}$ for $1 \mathrm{~h}$. The homogenate supernatants from each of the $\mathrm{E}$ and $\mathrm{C}$ rats were pooled separately.

Recipient rats ( 21 from four litters of the same age as the donors) had electrodes implanted unilaterally in the amygdala and were used 6 or more days later. Each recipient was lightly anesthesized with ether and received approximately $5 \mathrm{ml}$ of supernatant (the equivalent of two brains) via an intraperitoneal (IP) injection. Two groups of recipients were obtained: E, injected with supernatant from $\mathrm{E}$ donors, and $\mathrm{C}$, injected with supernatant from $\mathrm{C}$ donors. A third group received saline injections (S). E, C, and S rats were littermates. All recipients were coded and run by an experimenter at 100 microA $24 \mathrm{~h}$ later, and thereafter at approximately 12-h intervals, until six trials of CC resulted.

The results are shown in Table 1 for the dependent variables: number of trials to first $\mathrm{CC}$, number of trials to first of two consecutive BA.

An analysis of variance statistical procedure indicated that the recipients of supernatant from kindled animals showed a significantly greater mean number of trials to CC than did the two control groups $(F=5.61 ; d f=1,18$, $\mathrm{p}<.05)$. Significant differences did not occur when $\mathrm{C}$ was compared to $\mathrm{S}$. These results are consistent with those in the preliminary work (Gaito et al, 1973).

Similar results occurred with the BA data. E rats required a significantly greater number of trials prior to the first appearance of a definite behavioral automatism than did $\mathrm{C}$ and $\mathrm{S}$ rats $(\mathrm{F}=5.00, \mathrm{df}=1,18, \mathrm{p}<.05)$.

\section{Experiment 2}

The procedures were similar to those in Experiment 1, except that the research was conducted in two phases. In the first phase, six recipients were injected with supernatant from two brains of kindled rats $(E)$, and three others were injected with two-brain supernatant from nonkindled animals (C). The second phase used seven $\mathrm{E}$ and seven $\mathrm{C}$ recipients, each receiving supernatant from one brain in the IP injection.

In the first part, the nine reached the $\mathrm{CC}$ stage very quickly, such that 'any potential differences were obscured. The mean number of trials to the first CC (the main dependent variabie) was 5.8 for the E group and 7.3 for the $C$ group. The means in the second phase were: 18.4 for $\mathrm{E}$ and 11.3 for $\mathrm{C}$. These differences were not statistically significant $(p>.05)$. When the results were combined and analyzed by a variance analysis (Table 1), $\mathrm{E}$ and $\mathrm{C}$ groups were not differentiated $(p>.05)$. The BA data also showed no significant differences between $\mathrm{E}$ and $\mathrm{C}$.

\section{Experiment 3}

Ten litters of six rats each were used. The first two animals in each litter were donors: one $\mathrm{E}$ (stimulated) and one $\mathrm{C}$ (nonstimulated). After $10 \mathrm{CC}$ trials for each $E$, the $E$ and $C$ rats were sacrificed. The supernatant of each of the $10 \mathrm{E}$ donors was injected IP into a littermate. The same procedure was followed with the $10 \mathrm{C}$ donors. The fifth and sixth member of each litter received an injection from a recipient of Experiment 2, who thereby acted as donor for Experiment 3 . The recipient donors had six trials $\mathrm{CC}$ in Experiment 2. They received four more and were then sacrificed. Because of 
Table 1

Data for Recipients in Experiments I, II, and III

\begin{tabular}{llrcc}
\hline & & & Mean & Mean \\
& & No. & 1st CC* & 1st BA* \\
\hline \multirow{3}{*}{ Experiment I } & E & 12 & 13.3 & 7.3 \\
& C & 5 & 5.4 & 3.3 \\
& S & 4 & 7.8 & 3.8 \\
\multirow{3}{*}{ Experiment II } & E & 13 & 12.6 & 4.7 \\
& C & 10 & 10.1 & 3.9 \\
& E & 27 & 12.8 & 6.7 \\
& C & 9 & 12.9 & 5.9 \\
\hline
\end{tabular}

${ }^{*}$ Significant differences, $p<.05$, for comparison of $E$ with $C+S ; E=$ recipients injected with material from donors which had convulsed, $C=$ injection from nonstimulated donors, and $S=$ injection of saline.

the loss of some rats, there were 36 recipients: 9 receiving $\mathrm{E}$ injections, 9 receiving $\mathrm{C}$ injections, and 18 receiving injections from recipient donors.

The results are indicated in Table 1 . No significant differences resulted in either the $\mathrm{CC}$ or BA data.

The results of the three experiments suggested a retarding effect when a two-brain injection of supernatant from kindled rats was used, but no effect when one brain was employed. Thus an analysis was performed on all rats receiving two-brain injections (Experiment 1, first phase of Experiment 2, and the preliminary experiment $-10 \mathrm{E}$ and $5 \mathrm{C}$ rats, means of 22 and 12 , respectively, for first trial of $\mathrm{CC}$ ). A similar analysis was used for those involved with injections from one brain (Phase 2 of Experiment 2, Experiment 3). For the former, the $28 \mathrm{E}$ rats had a mean of 14.6 and the $13 \mathrm{C}$ animals, a mean of 8.4. This difference allowed for the rejection of the null hypothesis of no differences $(F=4.47, \mathrm{df}=1,39, \mathrm{p}<.05)$. In the one-brain analysis, the mean number of trials for the first $\mathrm{CC}$ was 13.9 for $34 \mathrm{E}$ rats and 12.2 for $16 \mathrm{C}$ animals, a difference which was not statistically significant.

Similar analyses were performed with the BA data. E was greater than $C(9.1$ vs 5.2$)$ in the two-brain-per-injection situation, but this difference was not significant because of greater individual differences in this data than is the case with CC data. The CC stage is more stereotyped and easier to identify than is the case for BA events. No significant differences resulted when one-brain data were analyzed (6.7 for $\mathrm{E}$ and 5.3 for $\mathrm{C}$ ).

\section{Experiments 4 and 5}

The results of these experiments seemed to indicate that the supernatant fraction from kindled animals predisposes naive recipients to reach the $\mathrm{CC}$ stage, and possibly the BA stage, in the kindling process at a slower rate than is the case if this fraction from nonkindled animals is used, as long as the IP injection consists of at least two-brain amounts. Thus, two further experiments were conducted to evaluate this possibility.
In Experiment 4 and Experiment 5, each rat was injected with the supernatant fraction equivalent to approximately two and one-half brains. The mean to first trial of $\mathrm{CC}$ was around 10 for the donors in these and the earlier experiments (Table 2), as was the case for the $\mathrm{C}$ group. The $\mathrm{CC}$ data for the recipients are shown in Table 3.

The mean for $\mathrm{E}$ was greater than for $\mathrm{C}$ in both experiments, but the differences were not statistically significant. However, when the two experiments were combined in a Littermates by Groups analysis, the difference was statistically significant $(F=5.00$, $\mathrm{df}=1,12, \mathrm{p}<.05)$. Likewise, when the data were analyzed for all rats receiving an injection containing two or more brain amounts (preliminary experiment and Experiments 1, 2, 4, and 5), the differences were statistically significant $(F=6.56, d f=1,66, p<.025)$. The means were: 14.3 for $42 \mathrm{E}$ rats and 8.9 for $26 \mathrm{C}$ rats. The corresponding means for the situation when one-brain amounts were used are: 13.9 for $34 \mathrm{E}$ rats and 12.2 for $16 \mathrm{C}$ rats; these means were not significantly different.

If all experiments are combined, the means are: 14.2 for $76 \mathrm{E}$ animals, 10.2 for $42 \mathrm{C}$ rats, 10.0 for 108 donor rats. (Having only 108 donors over all experiments might seem puzzling; however, some animals were used as recipients in one experiment and as donors in the next.) With such large numbers of animals, the difference between $\mathrm{E}$ and donors $+\mathrm{C}$ is obviously of a statistically significant nature $(\mathrm{F}=11.37, \mathrm{df}=1,223, \mathrm{p}<.001)$.

\section{DISCUSSION}

McIntyre and Goddard (1973) suggested a two-factor model to account for the intraanimal facilitation obtained when kindling the second amygdala and for the retardation that occurs when rekindling the first amygdala (plus an increased latency in both cases). These are: (a) a long-term effect of positive nature, possibly due to modified neural circuitry, (b) a short-term "aftereffect" of negative nature, which is almost completely dissipated by the end of 14 days.

Presumably, the (a) aspects can have an effect within the same animal but, if circuitry is involved, one would not expect positive transfer between animals (although chemicals involved in the modification of circuits might be transferred). However, the (b) aspect may be a chemical event and may be capable of being transferred

Table 2

Mean Number of Trials to First CC for Donors

\begin{tabular}{lrr}
\hline Experiment & No. & Mean \\
\hline I & 24 & 10.6 \\
II & 19 & 10.9 \\
III & 28 & 8.6 \\
IV & 16 & 10.8 \\
V & 21 & 10.0 \\
Combined & 108 & 10.0 \\
\hline
\end{tabular}


Table 3

Data for Recipients in Experiments IV and V

\begin{tabular}{|c|c|c|c|}
\hline & & No. & $\begin{array}{l}\text { Mean } \\
1 \text { st CC }\end{array}$ \\
\hline Experiment IV & $\begin{array}{l}\mathrm{E} \\
\mathrm{C}\end{array}$ & $\begin{array}{l}7 \\
6\end{array}$ & $\begin{array}{r}13.0 \\
8.5\end{array}$ \\
\hline Experiment $\mathrm{V}$ & $\begin{array}{l}E \\
C\end{array}$ & $\begin{array}{l}7 \\
7\end{array}$ & $\begin{array}{l}13.1 \\
10.4\end{array}$ \\
\hline Combined* & $\begin{array}{l}\mathrm{E} \\
\mathrm{C}\end{array}$ & $\begin{array}{l}14 \\
13\end{array}$ & $\begin{array}{r}13.1 \\
9.5\end{array}$ \\
\hline
\end{tabular}

*Significant differences, $p<.05$.

to other animals. Thus the present experiments seem to suggest that the "aftereffect" of McIntyre and Goddard may be that which is causing the retarding effect in recipients.

Assuming that this retarding effect is a genuine one (as these data suggest), a number of important questions may be raised. What is the specific molecular species which are responsible for the effect? Is it unique to the brain (rather than being distributed throughout the body)? If unique to the brain, is it localized in specific sites? Research is anticipated, or underway, relative to these questions.

Another important question concerns the retarding effect on recipients. Does it affect all recipients but vary in the degree of its effects? If so, the retarding effect would be superimposed on the natural susceptibility of the rats to convulse in response to the brain stimulation. Or does it affect only some animals? This latter possibility, a selective effect, may be the true state of affairs. The retarding effect observed when injections contained amounts equivalent to two or more brains was not a great one, and in each experiment there were a few $E$ rats who required 20 or more trials to reach the $\mathrm{CC}$ stage. There tended to be more of these late kindlers among the $\mathrm{E}$ group than with the donors or $\mathrm{C}$ recipients.

\section{REFERENCES}

Gaito, J. Molecular psychobiology. Springfield, Ill: Thomas, 1966.

Gaito, J. DNA complex and adaptive behavior. Englewood Cliffs, N.J: Prentice-Hall, 1971.

Gaito, J. Macromolecules and brain function. In J. Gaito (Ed.), Macromolecules and behavior. (2nd ed.) New York: A ppleton-Century-Crofts, 1972.

Gaito. J.. Hopkins, R. W., \& Pelletier, W. Interanimal transfer and chemical events underlying the kindling effect. Bulletin of the Psychonomic Society, 1973, 1, 319-321.

Goddard, G. V., McIntyre, D. C., \& Leech, C. K. A permanent change in brain function resulting from daily electrical stimulation. Experimental Neurology, 1969, 25, 295-330.

McIntyre, $D$. C. Differential amnestic effect of cortical vs amygdaloid elicited convulsions in rats. Physiology \& Behavior, 1970, 5, 747-753.

McIntyre, D. C., \& Goddard, G. V. Transfer, interference and spontaneous recovery of convulsions kindled from rat amygdala. Electroencephalography \& Clinical Neurophy siolog y, $1973,35,533-543$.

Racine, R. J. Modification of seizure activity by electrical stimulation: II. Motor seizure. Electroencephalography \& Clinical Neurophysiology, 1972, 32, 281-294.

(R eceived for publication September 20, 1973; revision received May 23,1974 .) 